\title{
CONTRIBUTIONS TO THE GENERAL THEORY OF TRANSFORMATIONS OF NETS*
}

BY

\author{
V. G. GROVE
}

\section{INTRODUCTION}

Let there be given a surface $S_{y}$ in projective space of three dimensions. Suppose that on $S_{y}$ we have two one-parameter families of curves such that through each point of $S_{\nu}$ there passes one curve of each family, the two tangents being distinct. Such a set of curves will be called a net $N_{y}$. Suppose that through each point $y$ of $S_{y}$ there passes a line $g$ of a congruence $G$, such that the developables of the congruence $G$ intersect $S_{\nu}$ in the curves of the given net $N_{y}$. Let $S_{z}$ be another surface in the same projective three-space, in one-to-one point correspondence to $S_{\nu}$, corresponding points lying on the lines $g$ of $G$. The developables of $G$ intersect $S_{z}$ in a net $N_{z}$. If neither $N_{y}$ nor $N_{z}$ is a focal surface of $G$, the nets $N_{\nu}$ and $N_{z}$ have been called nets in relation $C$ or $C$ transforms. $\dagger$ If the nets $N_{\nu}$ and $N_{z}$ are conjugate nets in relation $C$, they are in the relation of a transformation $F$.

In case $N_{y}$ and $N_{z}$ are $F$ transforms, the cross ratio $C$ formed by the pair of corresponding points and the pair of focal points on the line joining them is a projective invariant. $\ddagger$ In case $N_{\nu}$ and $N_{z}$ are $C$ transforms, there exists, of course, a similar invariant, $\S$ which we have denoted by $R$. It is the purpose of this paper to show that some of the theorems concerning the invariant $C$ related to the transformation $F$ are capable of generalization to the transformation $C$ and the associated invariant $R$. We also present a generalization of the transformation $\Omega$ and its associated invariant $H$ to our extended class of nets.

Without loss of generality we may assume that $N_{y}$ and $N_{z}$ are parametric. Let the parametric equations of $S_{y}$ and $S_{z}$ be

$$
y^{(k)}=y^{(k)}(u, v), \quad z^{(k)}=z^{(k)}(u, v) \quad(k=1,2,3,4)
$$

* Presented to the Society, September 6, 1928; received by the editors September 12, 1928, and (revised) May 28, 1929.

$\dagger$ V. G. Grove, Transformations of nets, these Transactions, vol. 30 (1928), p. 483. Hereafter referred to as Grove, Transformations.

$\ddagger$ W. C. Graustein, An invariant of a general transformation of surfaces, Bulletin of the American Mathematical Society, vol. 30 (1928), pp. 122 ff.

\& Grove, Transformations, p. 493. 
respectively. The four pairs of functions $(y, z)$ satisfy a system of differential equations of the form*

$$
\begin{array}{rrr}
y_{u u} & =\alpha y_{u}+\beta y_{v}+p y+L z, \\
y_{u v} & =a y_{u}+b y_{v}+c y+M z, \\
y_{v v} & =\gamma y_{u}+\delta y_{v}+q y+N z, \\
z_{u} & =m y_{u} & +f y+A z, \\
z_{v} & = & n y_{v}+g y+B z .
\end{array}
$$

We shall denote the coefficients of the differential equations which $y$ and $z$ satisfy, when their rôles in the formulation of system (1) are interchanged, by the corresponding primed letters. We shall refer the reader to the paper just cited for their values $\dagger$ in terms of the coefficients of system (1).

The coefficients $\alpha, \beta, \gamma$, etc. satisfy certain integrability conditions of which we use the following:

$$
\begin{aligned}
m_{v}+a(m-n)-g & =A m, \\
n_{u}+b(n-m)-f & =B n, \\
B_{u}-A_{v}+(n-m) M & =0 .
\end{aligned}
$$

From the integrability conditions, Green has shown that the following relation holds:

$$
(a+\delta+B)_{u}=(b+\alpha+A)_{v} .
$$

A similar formula exists for the primed letters arising when the rôles of $y$ and $z$ are interchanged. If we make the transformation

wherein

$$
y=\lambda \bar{y}, \quad z=\mu \bar{z}
$$

$$
\begin{gathered}
4 \frac{\partial}{\partial u} \log \lambda=b+\alpha+A, \quad 4 \frac{\partial}{\partial v} \log \lambda=a+\delta+B, \\
\mu=(m n)^{1 / 3}
\end{gathered}
$$

* Grove, Transformations, p. 484.

† On account of the difficulty of printing the symbols used in the previous papers, we have changed our notation. In order to enable the reader to make the change readily, we give the following transformation scheme:

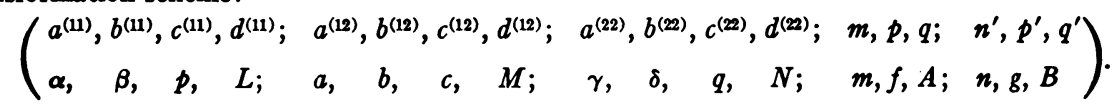

In case the letters of the first row are Greek, the corresponding letters below are primed. 
the resulting system of differential equations satisfied by $\bar{y}$ and $\bar{z}$ will be such that

$$
a+\delta+B=\alpha+b+A=a^{\prime}+\delta^{\prime}+B^{\prime}=\alpha^{\prime}+b^{\prime}+A^{\prime}=0 .
$$

We shall hereafter assume that the two systems are such that equations (4) are satisfied.

2. The GEOMETRIC ADJOINED SYSTEM OF DIFFERENTIAL EQUATIONS

The coördinates of the tangent planes to $S_{y}$ and $S_{z}$ at corresponding points $y$ and $z$ may be taken to be

$$
Y=\left|y, y_{u}, y_{v}\right|, \quad Z=\left|z, z_{u}, z_{v}\right|
$$

respectively. The system of differential equations of the form (1) satisfied by the four pairs of functions $(Y, Z)$ has the coefficients $\alpha^{\prime \prime}, \beta^{\prime \prime}, \gamma^{\prime \prime}$, etc. defined by the formulas

$$
\begin{aligned}
d \alpha^{\prime \prime} & =B^{\prime} L M+M\left(\alpha M-a L+M_{u}\right)-N\left(b L-\beta M+L_{u}\right)-A^{\prime} L N \\
d \beta^{\prime \prime} & =A^{\prime} L M+M\left(b L-\beta M+L_{u}\right)-L\left(\alpha M-a L+M_{u}\right)-B^{\prime} L^{2}, \\
d a^{\prime \prime} & =B^{\prime} M^{2}+L(a N-\gamma M)-N L_{v}+M\left(b N-\delta M+M_{v}\right)-A^{\prime} M N, \\
d b^{\prime \prime} & =A^{\prime} M^{2}+N(b L-\beta M)-L N_{u}+M\left(a L-\alpha M+M_{u}\right)-B^{\prime} L M, \\
d \gamma^{\prime \prime} & =B^{\prime} M N+M\left(a N-\gamma M+N_{v}\right)-N\left(\delta M-b N+M_{v}\right)-A^{\prime} N^{2}, \\
d \delta^{\prime \prime} & =A^{\prime} M N+M\left(\delta M-b N+M_{v}\right)-L\left(a N-\gamma M+N_{v}\right)-B^{\prime} L N \\
L^{\prime \prime} & =-L /(m n), \quad M^{\prime \prime}=-M /(m n), \quad N^{\prime \prime}=-N /(m n), \\
d m^{\prime \prime} & =n N L^{\prime}-m M M^{\prime}, \quad d t^{\prime \prime}=n N M^{\prime}-m M N^{\prime}, \\
d s^{\prime \prime} & =m L N^{\prime}-n M L^{\prime}, \quad d n^{\prime \prime}=m L N^{\prime}-n M M^{\prime}, \\
d f^{\prime \prime} & =-A m^{\prime \prime}-B s^{\prime \prime}, \quad d g^{\prime \prime}=-B n^{\prime \prime}-A t^{\prime \prime} \\
A^{\prime \prime} & =-A^{\prime}, \quad B^{\prime \prime}=-B^{\prime}, \quad d=M^{2}-L N .
\end{aligned}
$$

The system with coefficients (5) will be called the geometric adjoint of system (1).

We have called nets $N_{y}$ and $N_{z}$ in relation $L$, or $L$ transforms, if they are in relation $C$, and if the developables of the congruence of lines of intersection of corresponding tangent planes to the sustaining surfaces correspond to these nets. The conditions that $N_{y}$ and $N_{z}$ in relation $C$ be $L$ transforms $\operatorname{are}^{*}$

$$
m M N^{\prime}-n M^{\prime} N=n L^{\prime} M-m L M^{\prime}=0 .
$$

Let us interpret $Y$ and $Z$ as points instead of planes and denote by $N_{Y}$

* Grove, Transformations, p. 487. 
and $N_{z}$ the nets generated by them as $y$ varies over $S_{y}$. We see from (5) that if the nets $N_{y}$ and $N_{z}$ are in relation $L$, the nets $N_{Y}$ and $N_{Z}$ are in relation $C$. Similarly we may verify that if $N_{Y}$ and $N_{Z}$ are in relation $L$, the nets $N_{y}$ and $N_{z}$ are in relation $C$. The transformations $C$ and $L$ present a complete duality among the elements involved.

Suppose the net $N_{Y}$ has equal point invariants of the first kind.* We shall say in this case that $N_{y}$ has equal tangential invariants of the first kind. The net $N_{y}$ has equal tangential invariants of the first kind if and only if

$$
\left(a^{\prime \prime}-\gamma^{\prime \prime} M^{\prime \prime} / N^{\prime \prime}\right)_{u}-\left(b^{\prime \prime}-\beta^{\prime \prime} M^{\prime \prime} / L^{\prime \prime}\right)_{v}=0 .
$$

If use be made of formulas (5), equation (7) may be written in the following form:

$$
(a-\gamma M / N)_{u}-(b-\beta M / L)_{v}-\frac{\partial^{2}}{\partial u \partial v} \log \left(\frac{L}{N}\right)=0 .
$$

Hence if $N_{y}$ has equal point and equal tangential invariants of the first kind it follows that

$$
\frac{\partial^{2}}{\partial u \partial v} \log \left(\frac{L}{N}\right)=0 .
$$

Similarly if $N_{z}$ has equal point and equal tangential invariants of the first kind

$$
\frac{\partial^{2}}{\partial u \partial v} \log \left(\frac{L^{\prime}}{N^{\prime}}\right)=0 \text {. }
$$

Suppose now that $N_{y}$ and $N_{z}$ are non-conjugate nets in relation $L$. It follows from (6) that

$$
L^{\prime} / N^{\prime}=m^{2} L /\left(n^{2} N\right) .
$$

Hence if one of the non-conjugate nets $N_{y}$ and $N_{z}$ in relation $L$ has equal point and equal tangential invariants of the first kind, the other will have the same property only if $L$ is the transformation $\dagger K_{R}$.

\section{The transformation $\Omega_{H}$. The invariant $H$}

Suppose that $N_{y}$ and $N_{z}$ are in relation $L$. From (5) it follows that the focal planes of the line $h$ of intersection of the tangent planes to $S_{y}$ and $S_{z}$ at $y$ and $z$ form with these tangent planes the cross ratio

$$
H=m^{\prime \prime} / n^{\prime \prime}=n L^{\prime} N /\left(m L N^{\prime}\right),
$$

* V. G. Grove, Nets with equal $W$ invariants, in the present number of these Transactions, pp. 845-852.

† Grove, Transformations, p. 495. 
whatever the character of the nets $N_{y}$ and $N_{z}$, provided of course neither is asymptotic. We shall say that $N_{y}$ and $N_{z}$ are in the relation of a transformation $\Omega_{H}$ if they are in relation $L$ and if

$$
(\log H)_{u v}=0 \text {. }
$$

If $N_{y}$ and $N_{z}$ are conjugate nets in relation $\Omega_{-1}$ they are $\Omega$ transforms in the sense of Eisenhart.*

Consider now the product of the invariants $\dagger R=m / n$ and $H$. This product will be unity if and only if

$$
L / N=L^{\prime} / N^{\prime} \text {. }
$$

We have therefore the theorems of Slotnick $\ddagger$ : If two conjugate nets are in relation $F$, and the product of the invariants $R$ and $H$ is unity, the asymptotic nets on the sustaining surfaces correspond and conversely. And if one of two conjugate nets in relation $F$ is isothermally conjugate, the other is also if the product of $R$ and $H$ is unity.

Suppose $N_{y}$ and $N_{z}$ are non-conjugate and in relation $L$. From (6) we have

$$
M^{\prime} / N^{\prime}=m M /(n N) \text {. }
$$

If $R H=1$, it follows from (12) that

Hence

$$
M^{\prime} / L^{\prime}=m M /(n L)=n M /(m L) .
$$

$$
m^{2}-n^{2}=0 .
$$

Therefore if the product of the invariants $R$ and $H$ of non-conjugate nets in relation $L$ is unity, the nets are either radial transforms or $K_{-1}$ and $\Omega_{-1}$ transforms. In this case the asymptotic net on $S_{y}\left(S_{z}\right)$ corresponds to the harmonic reflection of that net in the parametric tangents on $S_{y}\left(S_{z}\right)$. Conversely if the sustaining surfaces of non-conjugate nets in relation $L$ are such that their asymptotics correspond to their harmonic reflections $\$$ in the tangents to the curves of the given nets, the product of $R$ and $H$ is unity. The transformation $L$ is therefore $\Omega_{-1}$.

* Eisenhart, Transformations of Surfaces, Princeton University Press, p. 134.

$\dagger$ Grove, Transformations, p. 493.

$\ddagger$ M. M. Slotnick, $A$ contribution to the theory of fundamental transformations of surfaces, these Transactions, vol. 30 (1928), p. 201. Hereafter referred to as Slotnick, Transformations.

$\S$ If it is understood that the harmonic reflection of the asymptotic tangents in the tangents to the curves of a conjugate net are these asymptotic tangents, the word "non-conjugate" may be deleted from these statements. 


\section{TRIADS OF NETS}

Let $N_{y}$ and $N_{z}$ be nets in relation $C$. Define the net $N_{\eta}$ by the formula

$$
\eta=\theta y_{u}+\phi y_{v}+z .
$$

One may readily verify that $N_{\eta}$ will be in relation $C$ to $N_{\nu}$ if and only if the functions $\phi$ and $\theta$ satisfy the following system of differential equations:

$$
\begin{aligned}
& \theta \beta+\phi b+\phi_{u}-\phi(\theta L+\phi M+A)=0, \\
& \phi \gamma+\theta a+\theta_{u}-\theta(\phi N+\theta M+B)=0 .
\end{aligned}
$$

Suppose that we have three nets $N_{y}, N_{z}, N_{\eta}$ so related that $N_{y}$ and $N_{z}$; $N_{z}$ and $N_{\eta} ; N_{\eta}$ and $N_{\nu}$ are $C$ transforms in the pairs indicated,by means of different congruences $G$ but the same congruences $H$. Such a triad of nets will be said to form an $H$ triad. If the nets of an $H$ triad are conjugate, the $H$ triad is an harmonic triad of conjugate nets in the sense of Slotnick.*

Let the transformations $C$ transforming $N_{y}$ into $N_{z} ; N_{z}$ into $N_{\eta}$; and $N_{\eta}$ into $N_{y}$ be denoted by $C_{1}, C_{3}$ and $C_{2}$ respectively. We shall speak of the transformation $C_{2}$ as the product of the transformations $C_{1}$ and $C_{3}$ and write

$$
C_{2}=C_{1} C_{3} \text {. }
$$

The coördinates $y, z, \eta$ of corresponding points of an $H$ triad satisfy the equations of the form

$$
\begin{array}{ll}
z_{u}=m_{1} y_{u}+f_{1} y+A_{1} z, & z_{v}=n_{1} y_{v}+g_{1} y+B_{1} z ; \\
\eta_{u}=m_{3} z_{u}+f_{3} z+A_{3} \eta, & \eta_{v}=n_{3} z_{v}+g_{3} z+B_{3} \eta \\
\eta_{u}=m_{2} y_{u}+f_{2} y+A_{2} \eta, & \eta_{v}=n_{2} y_{v}+g_{2} z+B_{2} \eta .
\end{array}
$$

From equations (13) we may derive the following relations:

$$
f_{1} / m_{1}=f_{2} / m_{2} ; \quad g_{1} / n_{1}=g_{2} / n_{2} ; \quad A_{1}=-f_{3} / m_{3} ; \quad B_{1}=-g_{3} / n_{3} .
$$

Hence if neither of the transformations $C$ giving rise to an $H$ triad is radial, and if one of the nets of the $H$ triad is conjugate, the other two nets of the triad have the same property.

Let $R_{1}, R_{2}, R_{3}$ be the three invariants $R$ of the corresponding transformations $C_{1}, C_{2}, C_{3}$ giving rise to an $H$ triad. From the relations existing between the coefficients of equations (13), one may readily prove that $R_{1} R_{3}=R_{2}$. If the transformations $C_{i}$ are each also transformations $L$, we may prove that $H_{1} H_{3}=H_{2}$, wherein $H_{1}, H_{2}$ and $H_{3}$ are the invariants $H$ of the transformations $C_{1}, C_{2}$ and $C_{3}$ respectively.

We may define also a $C$ triad as a set of three nets $N_{y}, N_{z}$ and $N_{\eta}$ such that

* Slotnick, Transformations, p. 195. 
they are in relation $C$ by means of the same congruence $G$ but by different congruences $H$. We may prove that the invariants $R_{i}$ and $H_{i}$ of nets of a $C$ triad satisfy the equation $R_{1} R_{3}=R_{2}$, and, if the transformations $C_{i}$ are also transformations $L$, that $H_{1} H_{3}=H_{2}$. We may state our results as follows: If the product of two transformations $L$ is a transformation $L$ the three nets in question form either a $C$ or an $H$ triad. In either case the invariants $R$ and $H$ of the product transformation are equal respectively to the products of the invariants $R$ and the products of the invariants $H$ of the two transformations.

Other theorems similar in character to those arising in the theory of $F$ transformations readily suggest themselves. We shall not enter into these details at present.

\section{RANGeS OF NETS IN RELATION $C$}

Consider a net $N_{z}$ in relation $C$ to the net $N_{y}$ by means of a congruence $G$. Let $N_{\zeta}$ be any other net in relation $C$ to $N_{\nu}$ by means of the same congruence $G$. The net $N_{\zeta}$ is defined by the expression

$$
\zeta=z-\lambda y,
$$

where $\lambda$ is a given arbitrary function of $u$ and $v$. The one-parameter family of nets $N_{\zeta(h)}$ in relation $C$ to $N_{y}$ defined by

$$
\zeta(h)=z-h \lambda y,
$$

where $h$ is a parameter independent of $u$ and $v$, will be called $a$ range of nets in relation $C$ to $N_{y}$.

As may easily be verified, the line of intersection of the tangent planes to the surfaces $S_{y}$ and $S_{\zeta}$ at corresponding points $y$ and $\zeta$ intersect in the line joining the points

$$
r^{\prime}=r+\frac{\lambda P}{m-\lambda} y, \quad s^{\prime}=s+\frac{\lambda P^{\prime}}{n-\lambda} y,
$$

where

$$
\begin{aligned}
r & =y_{u}+f y / m, & s & =y_{v}+g y / n, \\
P & =f / m+A-\lambda_{u} / \lambda, & P^{\prime} & =g / n+B-\lambda_{v} / \lambda .
\end{aligned}
$$

Hence the tangent planes to $S_{\nu}$ and $S_{\zeta(h)}$ intersect in the line joining

$$
r_{h}^{\prime}=r+\frac{h \lambda P}{m-h \lambda} y, \quad s_{h}^{\prime}=s+\frac{h \lambda P^{\prime}}{n-h \lambda} y .
$$

Referred to the tetrahedron $y, r, s, z$ the envelope of the lines joining the points (17) is the conic

(18) $\left[(m-n) x_{1}-n P x_{2}-m P^{\prime} x_{3}\right]^{2}+4 m(m-n) P^{\prime} x_{1} x_{3}=0, \quad x_{4}=0$. 
This conic is tangent to the parametric tangents at the points

$$
R=y_{u}+\left(\frac{f}{m}+\frac{n P}{m-n}\right) y, \quad S=y_{v}+\left(\frac{g}{n}+\frac{m P^{\prime}}{n-m}\right) y .
$$

Suppose that $N_{y}$ is not conjugate. The points $R$ and $S$ will coincide with the points of intersection* of the line $g^{\prime}$ in Green's relation $R$ to $g$ if and only if

$$
\begin{aligned}
A n+f+a(m-n)-n \lambda_{u} / \lambda & =0, \\
B m+g+b(n-m)-m \lambda_{v} / \lambda & =0 .
\end{aligned}
$$

By means of the integrability conditions (2), these equations reduce to

$$
\frac{\partial}{\partial u} \log \frac{\lambda}{n}=\frac{\partial}{\partial v} \log \frac{\lambda}{m}=0 .
$$

It follows therefore that

and

$$
(\log R)_{u v}=0
$$

$$
\lambda=c^{\prime} m=c n,
$$

where $c$ and $c^{\prime}$ are constants. Therefore $R$ is a constant. The nets $N_{y}$ and $N_{z}$ are consequently in relation $K_{R}$ where $R$ is a constant. From (22) it follows that

$$
\frac{m-\lambda}{n-\lambda}=\frac{m\left(1-c^{\prime}\right)}{n(1-c)} .
$$

Hence if the conic (18) is tangent to the parametric tangents at their intersection with the line $g^{\prime}$ in Green's relation $R$ to $g$, the range of nets $N_{\zeta(h)}$ is a range of nets in relation $K_{R}$ to $N_{y}$ where $R$ is a constant.

In case $N_{y}$ and $N_{\zeta(h)}$ are radial transforms, the lines of intersection of corresponding tangent planes are lines of a pencil with vertex

$$
P^{\prime} y_{u}-P y_{v}+\left(f P^{\prime} / m-g P / n\right) y \text {. }
$$

This point will be indeterminate if $P=P^{\prime}=0$. Under these conditions we have

$$
\lambda_{u} / \lambda=f / m+A=n_{u} / n, \quad \lambda_{v} / \lambda=g / n+B=m_{v} / m .
$$

Since the integrability condition of (23) is satisfied it follows that

where $c$ is a constant.

$$
\lambda=\mathrm{cm}
$$

* Grove, Transformations, p. 491.

Michigan State College, East Lansing, Mich. 